\title{
Multilinguales
}

\section{Le français au lycée en Algérie : des «types » de textes aux « genres » de discours}

French in High School in Algeria: from Text "Types" to Speech "Genres"

\section{Amar Ammouden}

\section{(2) OpenEdition}

1 Journals

Édition électronique

URL : http://journals.openedition.org/multilinguales/976

DOI : $10.4000 /$ multilinguales. 976

ISSN : 2335-1853

Éditeur

Université Abderrahmane Mira - Bejaia

Référence électronique

Amar Ammouden, "Le français au lycée en Algérie : des «types » de textes aux " genres » de discours », Multilinguales [En ligne], 6 | 2015, mis en ligne le 01 décembre 2015, consulté le 17 septembre 2019. URL : http://journals.openedition.org/multilinguales/976; DOI : 10.4000/multilinguales.976

Ce document a été généré automatiquement le 17 septembre 2019.

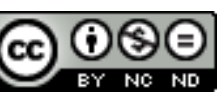

Multilinguales est mise à disposition selon les termes de la Licence Creative Commons Attribution Pas d'Utilisation Commerciale - Pas de Modification 4.0 International 


\title{
Le français au lycée en Algérie : des «types » de textes aux « genres » de discours
}

French in High School in Algeria: from Text "Types" to Speech "Genres"

\author{
Amar Ammouden
}

1 Les hypothèses que nous formulons pour le traitement de notre sujet sont les suivantes: c'est seulement au cours de cette dernière décennie que la notion du genre s'est frayé une place timide dans l'enseignement du français au lycée.

Les concepteurs de manuels n'ont pas émis de recommandations précises à ce sujet, même dans ceux où une entrée par les genres est retenue (cf. infra).

Enfin, l'observation des tables des matières des manuels nous autorise à penser qu'une confusion règne dans la grande majorité des manuels des trois dernières décennies au sujet de l'entrée par laquelle se fait l'apprentissage du français au secondaire (entrée par les genres ou entrée par les types de textes).

Cette contribution tentera donc d'apporter des éléments de réponse aux questions suivantes: Quelle évolution de l'enseignement du français au secondaire depuis les années 80 ? Quelle place pour l'enseignement par les genres de discours dans les manuels de français au secondaire? Les enseignants sont-ils conscients du passage d'une logique d'un enseignement par la typologie des textes à une logique d'enseignement par les genres de discours?

Cette prise de conscience étant nécessaire pour un apprentissage dont l'objectif principal est l'appropriation de la compétence communicative; d'où l'intérêt de la notion de " genre ». En effet, les interactions sociales se font par les genres de discours et non par les types de textes.

Dans cette étude, nous nous intéresserons à cette notion de genre et partant, à celle de la théorie des genres que nous détaillerons dans le premier point, avant de traiter, dans le second point, de l'évolution de l'enseignement/apprentissage du français dans le secondaire algérien par rapport à la place qu'il accorde à cette notion de genre de 
discours dans les pratiques de classe, et dans un troisième point, à l'attitude des enseignants par rapport à cette notion.

\section{Le passage du « type » au « genre »}

2 J. M. Adam (2005 : 13-14) fait le point sur cette notion de «type» en expliquant les raisons de son adoption dans les années 70 :

Les typologies de textes sont inséparables des grammaires de textes qui sont apparues dans les années 70, dans la mouvance et l'euphorie de la grammaire générative et transformationnelle. Avec les grammaires de textes, il s'agissait de produire les modèles génératifs de tous les textes bien formés de toutes les langues. A côté de l'universalité des grammaires de textes, les typologies de textes ont tenté de réduire la diversité des réalisations discursives et de proposer des grammaires spécifiques. (...) Les typologies de textes se sont développées dans le sillage des approches structurales du récit des années 1960-70. Elles ont ensuite profité des développements de la poétique et de la sémiotique de la description (travaux, en particulier, de Philippe Hamon), des travaux sur l'argumentation et sur l'explication (...), sur le dialogue et la conversation (...). C'est dans cette perspective que j'ai moi-même exploré cette piste de la fin des années 1970 au milieu des années 1980.

A partir des années 90 , la notion de type de texte a progressivement cédé la place à une classification par les genres (Adam, 2005 ; Bronckart, 2008; Chartrand, 2008 ; Reuter et al., 2010).

Jean-Michel Adam, qui a lui-même «exploré [la] piste » de la typologie des textes, en montre les limites et plaide pour une approche par les genres de discours, en se référant, entre autres, aux travaux de Mikhail Bakhtine sur les genres du discours ${ }^{1}:$ " On ne devrait parler ni de typologie de texte, ni de typologie de discours. Les typologies de discours doivent être remplacées par une réflexion sur les genres et la généricité. Les typologies de textes sont trop ambitieuses et impertinentes " (2005: 16).

Qu'entend-on par genre? Chartrand (2008: 264) propose une synthèse des recherches sur cette notion sous forme de définition:

Par genre, nous entendons une forme langagière orale ou écrite conventionnelle relativement stabilisée présentant des faisceaux de caractéristiques pragmatiques et discursives (situation de communication, but ou intention, univers représenté), textuelles (structuration, mode de mise en discours - appelé mode d'énonciation chez Bronckart, 2004 dominant : narration, description ...), linguistiques, graphiques et matérielles particulières.

Quant au rôle didactique que cette notion joue dans la communication langagière, B. Schneuwly, cité par Chartrand (2008: 264), affirme que "c'est au travers des genres que les pratiques langagières s'incarnent dans les activités des apprenants"; tandis que les types de textes «sont en rupture avec les représentations et les discours des apprenants (personne ne déclare lire ou écrire du "narratif" ou de l' "argumentatif") »(Reuter, 1996 : 31).

L'analyse des manuels scolaires algériens des trente dernières années nous permettra de retracer l'évolution de l'enseignement du français en Algérie durant cette période pour montrer la place qu'occupe l'apprentissage par les genres dans ces manuels. L'étude a porté sur douze (12) manuels des différents niveaux du secondaire, parus entre 1984 et 2015.

Pour l'appréciation de la prise de conscience par les enseignants du secondaire du passage d'une entrée par les types de textes à une entrée par les genres, nous avons distribué un questionnaire à quinze d'entre eux.

Ce questionnaire est précédé d'un tableau présentant les points essentiels de la table des matières du manuel de 3.A.S de $2004-2005$ et celui de $3^{\text {ème }}$.A.S. actuel (2007-2008). Nous précisons que la majorité des enseignants enquêtés a exercé au secondaire au 
moins au cours de la période allant de 2004 à 2008 et a donc utilisé les deux manuels cités.

\begin{tabular}{|l|l|}
\hline Manuel de $3^{\text {ème }}$ A.S. 2004-2005 & Manuel de 3 ${ }^{\text {ème }}$ A.S. 2007-2008 \\
\hline • Argumenter : Pour et contre & • Textes et documents d'histoire \\
\hline - Exhorter & $\cdot$ Le débat d'idées \\
\hline • Exposer & •L'appel \\
\hline - Raconter & $\cdot$ La nouvelle fantastique \\
\hline - Décrire & \\
\hline
\end{tabular}

Le choix de ces deux programmes se justifie par le fait qu'ils mettent justement en évidence le passage d'une approche par la typologie des textes à une approche par les genres. Les trois questions ouvertes que nous avons posées aux enseignants appellent des réponses qui devaient s'y référer :

1. Avez-vous constaté un changement dans le programme actuel de français en 3.A.S. par rapport au précédent?

2. Que pensez-vous de l'enseignement du français par les types de textes?

3. Selon vous, que doit-on enseigner en 3.A.S et comment devrait-on l'enseigner?

\section{Définition et pertinence de la notion de « genre »}

3 L'avantage de l'enseignement par les genres discursifs est d'abord de rapprocher les pratiques scolaires des pratiques sociales, puisque les genres appris à l'école sont directement investis dans les interactions de l'apprenant avec son milieu social, et ces interactions sont le sens du discours: "le terme de discours, tel qu'il apparait chez Benveniste (...), désigne la mise en cuvre du langage par des individus dans des situations concrètes » (Bronckart, 2004 : 102).

Pour établir une étroite relation entre la réalité langagière et les interactions sociales, Jean-Paul Bronckart substitue à la notion de discours celle d' " activité langagière » ou d'« agir langagier » (Bronckart, $2004: 102)$.

Par le recours à ces expressions, il distingue un «agir non verbal» de toutes les espèces, c'est-à-dire "tout comportement actif d'un organisme" (ibid.: 100), d'un "agir verbal » qui désigne «la mise en œuvre d'un agir communicatif verbal, mobilisant des signes organisés en textes » (ibid.), caractéristique de l'espèce humaine.

Ainsi, le "genre » est considéré comme un concept opératoire, du fait de son implication dans les interactions sociales quotidiennes.

Comme les genres de discours sont intimement liés aux activités langagières, et comme celles-ci sont illimitées, les genres de discours sont également innombrables. Par contre, les types de textes sont en nombre restreint (le texte narratif, le texte descriptif, le texte argumentatif, le texte explicatif ou informatif, le texte exhortatif ou injonctif et le texte conversationnel).

Mais un seul type de texte peut correspondre à un grand nombre de genres. A titre d'exemple, le type exhortatif peut convoquer les genres de l'appel, du règlement intérieur, de la recette de cuisine, de la consigne de sécurité, de la note de service, du mode d'emploi, de la règle de jeu, du panneau de signalisation routière, de la notice de 
médicament, du texte publicitaire, de l'horoscope, etc.; d'où d'ailleurs la non pertinence d'un classement par type ; car, s'agissant par exemple de genre de discours de la linguistique textuelle,

un texte à dominante narrative est généralement composé de relations d'actions, d'événements, de paroles et de pensées, il comporte des moments descriptifs et dialogaux plus ou moins développés. Parler, dans ce cas, de façon réductrice "de texte de type narratif " revient à gommer la complexité spécifique du texte en question. (Adam, 1999 a : 82)

Pour l'appréhension de cette "complexité spécifique, il a proposé la notion de « séquence » (1992 $2001: 30)$ :

Mon hypothèse est la suivante: les " types relativement stables d'énoncés » et les régularités compositionnelles dont parle Bakhtine sont à la base, en fait, des régularités séquentielles. Les séquences élémentaires semblent se réduire à quelques types élémentaires d'articulation des propositions. Dans l'état actuel de la réflexion, il me paraît nécessaire de retenir les séquences prototypiques suivantes: narrative, descriptive, argumentative, explicative et dialogale.

Jean-Michel Adam justifie l'abandon de la notion de «type » et le recours à la notion de séquence :

J'ai consacré plusieurs articles, entre 1987 et 1992, et un livre (1992) à tenter d'expliquer pourquoi il est, à mon sens, profondément erroné de parler de "types de textes". L'unité "texte" est trop complexe et trop hétérogène pour présenter des régularités linguistiquement observables et codifiables, du moins à ce niveau de complexité. C'est pour cette raison que j'ai, à la différence de la plupart de mes prédécesseurs anglo-saxons, proposé de situer les faits de régularité dits "récit", "description", "argumentation", "explication" et "dialogue" à un niveau moins élevé dans la complexité compositionnelle, niveau que j'ai proposé d'appeler séquentiel. (1999: 82)

Certes, la notion de séquence a été remise en cause par un certain nombre de chercheurs dont François Rastier (2001), quant à sa position par rapport au genre ${ }^{2}$.

Nous retenons pour notre part qu'elle constituait pour son auteur une réponse à la prise en charge de l'hétérogénéité des textes que la notion de type annihilait, à l'instar de la notion de genre dont Sophie Moirand a proposé une définition qui rend compte de sa complexité :

une définition toujours provisoire mais un peu plus précise du genre, qu'on considère comme une représentation socio-cognitive intériorisée que l'on a de la composition et du déroulement d'une classe d'unités discursives, auxquelles on a été "exposé" dans la vie quotidienne, la vie professionnelle et les différents mondes que l'on a traversés, une sorte de patron permettant à chacun de construire, de planifier et d'interpréter les activités verbales ou non verbales à l'intérieur d'une situation de communication, d'un lieu, d'une communauté langagière, d'un monde social, d'une société... (2003:20)3

Plus récemment, Jean-Jacques Richer (2011) ${ }^{4}$, après avoir écarté la typologie des textes et la notion de séquence (idem.),

lorsque l'on veut travailler la compréhension/ production de textes authentiques appréhendés dans leur entièreté, lorsque l'on veut doter les apprenants d'outils langagiers qui leur permettront d'appréhender les énoncés dans leur totalité sémantique, dans leur dimension pragmatique et, éventuellement, de produire des textes » (idem.),

et après avoir retracé l'évolution de la recherche sur la notion de genre depuis «Bakhtine-Medvedev-Volochinov », puis Petitjean (1991), Maingueneau (1996), jusqu’à Adam $(2001)^{5}$, va proposer une approche affinée par les genres :

À partir de ces différentes définitions du cadrage générique, j'ai proposé (Richer, 2005) de procéder à un regroupement des instructions véhiculées par un genre de discours selon les cinq niveaux constitutifs de la totalité textuelle que ces prescriptions affectent. (op. cit. : 19)

Ces « instructions sont au nombre de six : 1- la dimension matérielle (caractéristiques formelles du médium, dimension spatiale et/ou dimension temporelle), 2- la dimension 
socio-pragmatique (statut et rôle des coénonciateurs, identification du système d'énonciation global, identification de l'acte de parole global, dimension du logos, de l'éthos et du pathos), 3- la dimension textuelle (dimension du plan de texte et de la combinatoire de schémas séquentiels), 4- la dimension stylistique ( variété(s) de langue), 5- la dimension thématique, 6-la dimension culturelle (rajoutée plus tard) (idem.).

Cet historique, forcément bref et schématique, nous permet cependant d'établir un lien étroit entre la notion de genre et l'approche par compétence en vigueur actuellement dans l'enseignement en Algérie. En effet, comme l'enseignement par les genres, l'approche par compétence permet de lier les savoirs aux pratiques sociales.

\section{Le genre de discours dans les manuels de français du secondaire}

Dans l'enseignement du français en Algérie, l'entrée adoptée diffère d'une période à une autre, et parfois même d'un niveau à un autre durant la même période. C'est le cas, par exemple, du manuel de $1^{\text {ère }}$ année secondaire, paru en 1985 1986, qui adopte une entrée par la grammaire, et de celui de la $2^{\text {ème }}$ année secondaire, paru en 1984 1985, qui adopte une entrée par les thèmes.

De manière générale, on passe d'une approche par les intentions communicatives (raconter, décrire, argumenter, ...), à une approche par les types (le texte narratif, le texte argumentatif, ...), à une approche par les genres.

\section{Une entrée par la grammaire ou par le thème}

Le manuel "Recueil de textes et exercices de langue - $1^{\text {ère }}$ année secondaire », paru en 1985 1986, adopte une entrée par la grammaire. Il est scindé en cinq chapitres :

- le noyau prédicatif,

- le déterminant obligatoire du prédicat : le sujet,

- la qualification,

- les expansions du prédicat

- la fonction autonomisée (adverbes et circonstants de lieu et de temps).

Ce manuel comporte une série de textes, littéraires pour la plupart, qui « permettent une sensibilisation au point de langue et son identification " (MEN : 1985-1986 : 3).

L'approche par la grammaire qui est proposée ne diffère pas fondamentalement de la méthodologie traditionnelle; quant à l'approche par la typologie de textes, elle est annoncée dans la table des matières de ce manuel par ce que ses concepteurs nomment les techniques d'expression (le récit et la description).

Par contre, dans le manuel de la $2^{\text {ème }}$ année secondaire, intitulé «Recueil de textes et techniques d'expression", paru en 1984-1985, on préconise une entrée par le thème: l'école, la famille, l'éducation, la vie affective, le sport, les jeux, les problèmes sociaux et les mass médias. Chacun des textes est suivi d'un questionnaire comportant quatre parties : compréhension globale, compréhension approfondie, exploitation linguistique (morphosyntaxe/lexique) et prolongement écrit.

Le manuel de la $3^{\text {ème }}$ année secondaire "Recueil de textes ", paru en 1986-1987, obéit à la même logique que le manuel de la deuxième année que nous venons de citer. Cependant, les textes de celui-ci ne sont pas suivis de questionnaires de compréhension et les thèmes qui y sont abordés relèvent le plus souvent du domaine scientifique ou 
philosophique. Concernant le choix de ces textes, il est justifié dans la présentation du manuel :

Que les textes présentent des caractères d'abstraction et de généralité et une certaine affinité avec le programme de philosophie des Terminales est tout à fait naturel à ce niveau des études (...). Les intentions des auteurs seraient mieux perçues si on replaçait l'ensemble de l'ouvrage dans l'éclairage du chapitre initial de l'unité "Sémiotiques", chapitre où sont rassemblés quelques concepts opératoires de la linguistique et des sciences sociales. Il nous a semblé qu'au terme du cycle secondaire le recours à ces concepts familiers à une grande partie de notre population scolaire permettrait une prise de conscience des mécanismes de la communication linguistique et sociale, la synthèse critique des acquis des années antérieures et une forme de présentation aux études supérieures. (MEN, $19861987: 5$ )

Dans ce «chapitre où sont rassemblés quelques concepts opératoires de la linguistique et des sciences sociales (...) [et qui] permettrait une prise de conscience des mécanismes de la communication linguistique et sociale ", la notion de "genre " occupe de fait la première place puisque : «toute action langagière s'inscrit dans un secteur donné de l'espace social : une institution qui doit être pensée comme une formation sociodiscursive, c'est à dire comme un lieu social associé à des genres de discours » (Adam, 1999 : 86).

Si comme l'affirme Adam, le genre est consubstantiel au discours, y recourir pour le développement de la compétence de communication sur laquelle se fonde l'approche communicative en vigueur dans l'enseignement des langues durant cette période, aurait pu relever de l'évidence.

\section{Une entrée par les types de textes ou par les intentions communicatives}

Le manuel "Français $2^{\text {eme }}$ A.S. ", paru en1988 1989, semble amorcer un changement d'orientation par rapport au précédent, en adoptant une approche par la typologie de textes, ou plus précisément par les intentions communicatives (exposer, prescrire, démontrer, argumenter, exhorter).

En dépit de ce changement, les concepteurs de ce manuel ne se sont pas totalement détachés de l'ancienne approche par le thème. En effet, tous les supports de l'unité didactique portant sur le reportage, par exemple, traitent de la «beauté féérique » du désert. Le manuel commence par un reportage intitulé "Taghit l'enchanteresse " (oasis du sud algérien). Il précède un récit de voyage dont le titre est «Deux cents kilomètres dans le Ténéré du Niger ». Ces deux textes sont suivis de quelques exercices de vocabulaire relatif au désert, visant la familiarisation de l'apprenant avec des termes relatifs au désert : erg, reg, oasis, etc.

Pourtant, ce vocabulaire n'est pas ciblé dans la production finale pour laquelle il est demandé aux apprenants de rédiger le premier paragraphe d'un reportage sur un lieu touristique ou un court reportage sur un lieu historique, un monument ou un lycée.

Ce thème est également choisi pour l'étude de la technique portant sur la synthèse de documents qui succède à l'unité didactique sur le reportage, à travers des supports textuels comme "Célébration de la journée mondiale : sous le signe de la lutte contre l'avancée des déserts » (Actualité de l'émigration du 30.10.85), «La menace du désert » (El Moudjahid du 21.12.86) et « alerte aux déserts » (L'Education du 20.10.77).

Par ailleurs, tous les supports proposés pour la dernière unité didactique « exhorter », y compris les lectures complémentaires, ont un thème commun : la solidarité et la paix.

L'ouvrage intitulé "Livre de français - $2^{\text {ème }} A . S$. », paru plus d'une décennie plus tard, en 2004 2005, est construit sur le même modèle que le précédent. Il est organisé en cinq projets: le reportage, l'expositif, le prescriptif, le démonstratif et l'argumentatif. Certaines activités constituent une simple reprise du manuel de 19881989 : reprise du 
thème du désert, à travers un reportage sur la région du M'zab, et étude du vocabulaire du désert.

Il faut souligner que les deux manuels font appel aux deux approches : par les types et par les genres de textes, puisqu'ils commencent tous les deux par un projet portant sur le reportage, qui s'inscrit dans le genre de discours, suivis par d'autres projets (argumentatif, prescriptif, expositif, etc.) qui s'inscrivent dans la typologie des textes.

Il en est de même pour le manuel intitulé «Livre de français : Raconter, dialoguer, décrire $1^{\text {ère }}$ A.S. », paru en 1989 1990, dans lequel les types de textes, annoncés dans le titre, sont mêlés à des genres comme le «fait divers" et le "portrait»; et pour le manuel " Français $3^{\text {ème }}$ A.S. ", paru la même année que le précédent, et dans lequel des types de textes, comme « l'expositif » et « l'argumentatif » (exprimer son opinion - convaincre), sont associés à des genres de discours comme l'«article encyclopédique », le poème, le roman, l'exposé, etc.

Le manuel intitulé "Livre de français - $3^{\text {ème }}$ A.S. ", paru en 2004 2005, évite cette confusion grâce à sa focalisation sur la typologie des textes. Il comporte quatre projets: Argumenter : Pour et contre, exhorter, exposer, raconter - décrire.

\section{Une entrée par les genres de textes}

A partir de 2006, l'entrée par le genre de texte commence à poindre dans les intitulés des séquences didactiques du français dans le secondaire.

Ainsi, le programme de troisième année propose le document historique, l'appel, le débat d'idées, la nouvelle fantastique et le texte poétique. ses objectifs tels que formulés dans le préambule (programme de $3^{\text {ème }}$ A.S., septembre 2006) plaident en ce sens :

Le nouveau programme poursuit des objectifs qui dépassent le cadre de la classification typologique des textes et accorde une grande importance à la linguistique de l'énonciation qui pose comme préalable qu'il faut distinguer ce qui est dit (contenu du texte) de la présence de l'énonciateur dans son propre discours. Il adopte une démarche de projet pédagogique et vise l'installation de compétences par des activités variées qui permettront aux apprenants de s'approprier les règles régissant les différents discours et les enjeux qui les sous-tendent.

Nous pouvons identifier ici, une référence implicite à la conception de Maingueneau du "genre de discours » dans sa dimension pragmatique, c'est-à-dire, un "acte de langage d'un niveau de complexité supérieure" (1998: 51) ${ }^{6}$. Cet acte relève donc de genres différents auxquels l'élève devra être confronté dans le cadre d'une pédagogie de projet.

Dans celui de la deuxième année, l'étude de plusieurs genres est proposée, dont le plaidoyer et le réquisitoire, le discours théâtral, le reportage touristique/le récit de voyage, la nouvelle d'anticipation, etc. Les « techniques d'expressions » ciblées sont la prise de note, l'exposé oral, le compte rendu, la fiche de lecture, etc.

Il en est de même du programme de la première année qui comprend les genres comme les textes de vulgarisation scientifique, l'interview/l'entretien, la lettre ouverte, le fait divers, la nouvelle, les poèmes et les chansons. Les "techniques d'expression" proposées sont le plan, la prise de notes, le résumé, la lettre, l'exposé oral, le C.V., etc.

5 Nous pouvons constater que, même si les références théoriques qui sous-tendent ces programmes ne sont pas explicitement affichées, il n'en demeure pas moins qu'elles existent. Il en est ainsi de celle des genres tacitement reconduite dans l'enseignement $\mathrm{du}$ français (première) langue étrangère dans le sens que lui ont conféré J. Dolz et Schneuwly B. (1998: 64-65) : 
Les genres peuvent être considérés de ce point de vue comme des outils qui fondent la possibilité de communication (et d'apprentissage). (...) Comme dans toute action humaine, il va utiliser un outil - ou un ensemble d'outils - pour agir : une fourchette pour manger, une scie pour abattre un arbre; l'action de parler, elle, se réalise à l'aide d'un genre qui est un outil pour agir langagièrement. C'est un outil sémiotique constitué de signes organisés de manière régulière (...); mais fondamentalement il s'agit d'un outil qui permet de réaliser une action dans une situation particulière. Et apprendre à parler, c'est s'approprier des outils pour parler dans des situations langagières diverses, c'est-à-dire s'approprier des genres.

Cette conception du genre comme « outil pour parler dans des situations langagières diverses" est compatible avec les "objectifs du nouveau programme [qui a adopté la] "démarche de projet pédagogique et [qui] vise l'installation de compétences par des activités variées qui permettront aux apprenants de s'approprier les règles régissant les différents discours et les enjeux qui les sous-tendent » (cf. supra).

\section{Les enseignants du secondaire et les genres de discours}

Le substrat théorique quant à l'approche adoptée (approche par les genres ou approche par les types) étant généralement implicite dans les manuels et les programmes du secondaire, les réponses des enseignants du secondaire interrogés s'en ressentent.

7 A la question relative aux changements apportés par le nouveau manuel de $3^{\text {ème }}$ année de septembre 2006 par rapport à celui de 2004-2005, dans leurs réponses, les enseignants n'ont pas fait allusion à ce passage de la typologie des textes aux genres de discours, pourtant primordial au niveau de la méthodologie de l'enseignement. Certes, la majorité d'entre eux a perçu une nouveauté plutôt qu'un changement : l'introduction $\mathrm{du}$ document d'histoire et de la nouvelle fantastique, de quelques techniques d'expression, d'une nouvelle terminologie, de thèmes d'actualité, suppression de la grammaire du texte, moins d'activités grammaticales, etc.

Certains considèrent que ce nouveau programme n'a apporté aucun changement par rapport au précédent: «En fait, dans le programme précédent, on met en intitulé la compétence; alors que dans le programme actuel, ce sont les contenus qui sont ciblés mais pour les mêmes compétences ».

Mais une minorité a saisi l'enjeu du nouveau programme par rapport au précédent :

- "Il ya un changement car l'actuel programme s'appuie sur l'analyse du discours et la structuration textuelle en tant que croisement discursif obéissant à une situation de communication et une visée communicative en fonction desquelles s'opère l'intégration des différents discours (expliquer, argumenter, raconter, etc.)»;

- "le programme actuel n'est pas fondé sur la typologie des textes";

Ces réponses, dans toutes leurs nuances, sont le résultat de ce que les programmes et les avant-propos des manuels scolaires n'ont pas affirmé clairement le choix méthodologique en faveur de l'approche par les genres de textes, et qu'ils recourent indifféremment à la notion de type et à la notion de genre, sans précaution méthodologique.

8 Pour conclure, nous pouvons affirmer que durant cette dernière décennie (2006 - 2015), l'entrée par la typologie des textes a certes cédé progressivement la place à une entrée par les genres de discours. Toutefois, faute de recommandations clairement formulées dans les manuels et les documents d'accompagnement des programmes, la majorité des enseignants du secondaire n'ont pas mesuré ce passage de l'approche par la typologie 
des textes à l'approche par les genres de discours. Par conséquent, leurs pratiques de classe n'ont pas fondamentalement changé.

\section{BIBLIOGRAPHIE}

ADAM J. M., Linguistique textuelle. Des genres de discours aux textes, Nathan, coll. FAC, Paris, 1999.

ADAM J. M., Les textes : types et prototypes. Récit, description, argumentation, explication et dialogue, Éditions Nathan, Paris, 1992-2001.

ADAM J. M., « La notion de typologie de textes en didactique du français : une notion « dépassée »? ", in Recherches, Académie de Lille, n 42, 2005, pp. 11-23.

BAKHTINE M., Esthétique de la création verbale, Gallimard, Paris, 1984.

BRONCKART J-P., «Les genres de textes et leur contribution au développement psychologique », in Langages, $\mathrm{n}^{\circ}$ 38, 2004, pp. 98108.

CHARTRAND S-G., « Proposition didactique d'une progression des objets à enseigner en français langue première au secondaire ", dans Dolz J. et Simard C. (dirs), Pratiques d'enseignement grammatical, Presses de l'Université Laval, Québec, 2009.

CHARTRAND S-G., DE KONINCK G., « La clarté terminologique pour une plus grande cohérence et rigueur dans l'enseignement du français ", in Québec français, $n^{\circ} 153,2009$, p. 37-39. Disponible sur [Url] :

< http://www.erudit.org/culture/qf1076656/qf1097061/44246ac.pdf > Consulté en mai 2015.

DENIZOT N., « Classements en genres et autres classifications textuelles », in Recherches, $\mathrm{n}^{\circ} 42$, 2005, pp. 37-61.

DOLZ J. \& SCHNEUWLY B., Pour un enseignement de l'oral - Initiation aux genres formels à l'école, ESF éditeur, Paris, 1998, 2009 ( $4^{\text {ème }}$ éd.).

MOIRAND S., "Quelles catégories descriptives pour la mise au jour des genres du discours? ", Journée d'étude Les genres de l'oral, 18 avril 2003, Université Lumière Lyon 2. Consultable sur le site : < http://gric.univ-lyon2.fr/Equipe1/actes/Journee_Genre/Moirand_cat_genres.rtf >

PORTILLOS ERRANO V., « La notion de genre en sciences du langage ». Consultable sur le site : < http://www.revue-texto.net/docannexe/file/2577/genre_portillo.pdf >

PUREN C., « Domaines de la didactique des langues-cultures. Entrées libres », in Les Cahiers pédagogiques, nº 437, CRAP, Paris, 2005, pp. 41-44.

RASTIER F., Arts et sciences du texte, P.U.F., Paris, 2001.

REUTER Y., Enseigner et apprendre à écrire, ESF Éditeur, Paris, 1996.

REUTER Y., COHEN-AZRIA C., DAUNAY B., DELCAMBRE I. \& LAHANIER-REUTER D., Dictionnaire des concepts fondamentaux des didactiques, De Boeck., Bruxelles, 2010. 


\section{Corpus - Manuels de français du secondaire}

AMIR A., KHOUKHI H. ET MELZI A., Français, Première année secondaire Scientifique \& Technologique, ONPS, Alger, 2005-2006

BOUCHOUIKA F., CHABANE F., ATTATFA DJ., BEHLOULI A. et al., Français 3e A.S., IPN, Alger, 1989-1990.

BOUCHOUIKA F., CHABANE F., BEHLOULI A., KHICHANE T. et al., Livre de français - 3ème AS, ONPS, Alger, 2004-2005.

CHABANE F., DELLIDJ J., ATTATFA DJ., BEHLOULI A. et al., Français $2^{e}$ A.S., IPN, Alger,1988-1989.

CHABANE F., DELLIDJ J., ATTATFA DJ., BEHLOULI A. et al., Livre de français - $2^{e}$ A.S., ONPS, Alger, 2004-2005.

DELLIDJ J., GOUASMI F. Z., ATTATFA DJ., BEHLOULI A. et al., Livre de français - Raconter, dialoguer, décrire - $1^{\text {ère }}$ A.S., ONPS, Alger, 1989-1990.

DJILALI K., BOULTIF A., LEFSIH A., Français, Première année secondaire Lettres, ONPS, Alger, 2005

MAHBOUBI F., REKKAB M. ET ALLAOUI A., Français - Troisième Année Secondaire, ONPS, Alger, 2007-2008.

MAINGUENEAU, D., Analyser les textes de communication, Dunod, Paris, 1998.

MINISTERE DE L'EDUCATION NATIONALE, Recueil de textes et techniques d'expression - 2ème année secondaire, IPN, Alger, 1984-1985.

MINISTERE DE L'EDUCATION NATIONALE, Recueil de textes et exercices de langue - $1^{\text {ère }}$ année secondaire, IPN, Alger, 1985-1986.

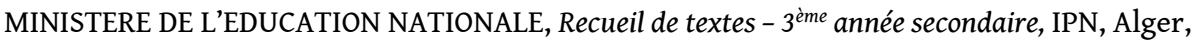
1986-1987.

ZEGRAR B., BOUMOUS A. ET BETAOUAF R., Français - Deuxième Année Secondaire, MEN/ONPS, Alger, 2006-2007.

\section{NOTES}

1. Bakhtine, M., Esthétique de la création verbale, Paris, Gallimard, 1979.

2. Pour la synthèse de la critique de cette notion, cf. Verónica Portillo Serrano, «La notion de genre en sciences du langage ». Consultable sur le site :

< http://www.revue-texto.net/docannexe/file/2577/genre_portillo.pdf >

3. "Quelles catégories descriptives pour la mise au jour des genres du discours?", Journée d'étude Les genres de l'oral 18 avril 2003, Université Lumière Lyon 2. Consultable sur le site :

http://gric.univ-lyon2.fr/Equipe1/actes/Journee_Genre/Moirand_cat_genres.rtf

4. Jean-Jacques Richer, «Les genres de discours : une autre approche possible de la sélection de contenus grammaticaux pour l'enseignement/ apprentissage du F.L.E. ?», Linx [En ligne], 64-65 | 2011, mis en ligne le 01 juillet 2014. URL : http://linx.revues.org/1396

5. "Les propositions de Bakhtine-Medvedev-Volochinov ont été approfondies, notamment dans le domaine francophone. (...) Ainsi A. Petitjean énumère sept prescriptions génériques en écrivant que les genres " réferent essentiellement: à l'ancrage institutionnel, à la situation de production et au matériau de réalisation, à l'intention communicationnelle, au mode énonciatif, à l'organisation formelle, au contenu thématique"(A. Petitjean, 1991: 352). D. Maingueneau (1996: 44) reconduit dans un premier temps, à quelques variantes près, les propositions de A. Petitjean lorsqu'il affirme que les "contraintes définitoires 
d'un genre (sont) : le statut respectif des énonciateurs et des coénonciateurs; les circonstances temporelles et locales de l'énonciation; le support et les modes de diffusion; les thèmes qui peuvent être introduits ; la longueur, le mode d'organisation, etc.";mais, deux ans plus tard, il souligne la dimension pragmatique d'un genre de discours en l'assimilant à un acte de langage: "Acte de langage d'un niveau de complexité supérieure, un genre de discours est soumis lui aussi à un ensemble de conditions de réussite" (1998: 51). Enfin, J.M. Adam (2001: 41) porte à huit les instructions textuelles que comporte un genre de discours en ouvrant le genre sur ses limites ("composante péritextuelle ") et sur son insertion dans des discours "méta" (" composante métatextuelle"): " une composante sémantique (...),une composante énonciative (...), une composante pragmatique (...), une composante stylistique et phraséologique (...), une composante compositionnelle (...),une composante matérielle (...), une composante péritextuelle (...), une composante métatextuelle (...)" » (J. J. Richer, idem.).

6. Conception décrite par J.-J Richer (cf. note 5 ici même).

\section{RÉSUMÉS}

A travers l'analyse de douze manuels de français au lycée, nous tenterons de souligner dans cet article les transformations opérées dans l'enseignement du français durant les trois dernières décennies. D'une part, nous nous interrogerons sur la place qu'occupe l'enseignement/ apprentissage par les genres de discours, fortement recommandé par les didacticiens à partir des années 90. D'autre part, et à l'aide d'un questionnaire destiné aux enseignants du secondaire, nous apporterons des éléments de réponse à la question sur la prise de conscience de ces enseignants du passage d'un enseignement par la typologie des textes à un enseignement par les genres de discours.

Through the analysis of twelve French textbooks in high school, we will try to highlight in this article the transformations of the teaching of French in the last three decades. We will focus in particular on the place of the teaching/learning by the speech genres, highly recommended by educationists from the 90 's. On the other hand, through the analysis of questionnaires désigned for secondary school teachers, we will try to discuss teachers attitudes towards this shift in teaching, that is from teaching language typology to teaching of discourse genres.

\section{INDEX}

Mots-clés : approche par genres de discours, approche par types de textes, programmes scolaires du FLE, manuels scolaires du secondaire

Keywords : speech genres based approach, texts types based approach, FFL school programs, secondary school textbooks

\section{AUTEUR}

\section{AMAR AMMOUDEN}

Faculté des lettres et des langues Laboratoire LAILEMM Université A. Mira - Bejaia Algérie 